\title{
Survival outcomes and prognostic factors of primary small cell carcinoma of the esophagus
}

\author{
Huikai Miao^, Rongzhen Li, Dongni Chen, Jia Hu, Youfang Chen, Zhesheng Wen \\ Department of Thoracic Oncology, State Key Laboratory of Oncology in South China, Collaborative Innovation Center for Cancer Medicine, Sun \\ Yat-sen University Cancer Center, Guangzhou, China \\ Contributions: (I) Conception and design: H Miao, R Li; (II) Administrative support: Z Wen; (III) Provision of study materials or patients: D Chen, J \\ Hu, Y Chen; (IV) Collection and assembly of data: H Miao, D Chen; (V) Data analysis and interpretation: H Miao, R Li; (VI) Manuscript writing: \\ All authors; (VII) Final approval of manuscript: All authors. \\ Correspondence to: Zhesheng Wen. Department of Thoracic Oncology, State Key Laboratory of Oncology in South China, Collaborative \\ Innovation Center for Cancer Medicine, Sun Yat-sen University Cancer Center, 651 Dongfengdong, Guangzhou 510060, China. \\ Email: wenzhsh@sysucc.org.cn.
}

Background: Primary small cell carcinoma of the esophagus (PSCCE) is a rare and aggressive malignancy. It has a poor survival rate, and there is no consensus as to a standard therapeutic modality. In this study, we aimed to investigate the prognostic factors and evaluate the outcomes of patients with PSCCE who had been treated with different therapeutic methods.

Methods: We retrospectively evaluated 113 consecutive patients with PSCCE who received treatment at our center from 2003 to 2016. The primary endpoint was overall survival (OS). The Cox regression model was used to analyze the prognostic factors. The survival analysis was calculated using the Kaplan-Meier and log-rank methods.

Results: The 12- and 36-month OS rates of all 113 enrolled patients were $45 \%$ and $12 \%$, respectively. A significantly prolonged OS rate was associated with lymph node stages N0-N1 $(\mathrm{P}=0.022)$, the Veterans' Administration Lung Study Group (VALSG) system limited-disease (LD) staging $(\mathrm{P}=0.040)$, and multimodality treatments $(\mathrm{P}=0.047)$. Patients with regional lymph node metastasis benefited more from surgery combined with chemotherapy than surgery or chemotherapy alone $(\mathrm{P}=0.046)$. Concerning chemotherapy, cisplatin plus etoposide was the regimen most commonly used to treat PSCCE patients $(67.5 \%)$.

Conclusions: An early lymph node stage, the VALSG LD staging, and multimodality treatments were identified as independent prognostic factors of PSCCE. Surgery combined with adjuvant chemotherapy was especially necessary for LD stage PSCCE patients with lymph node stages N1-3.

Keywords: Primary small cell carcinoma; esophagus; prognosis; treatment

Submitted Nov 19, 2020. Accepted for publication Mar 05, 2021.

doi: $10.21037 /$ jtd-20-3334

View this article at: http://dx.doi.org/10.21037/jtd-20-3334

\section{Introduction}

Esophageal carcinoma is one of the fastest-growing malignancies worldwide and the 5 th most common cancer in China (1). The number of new cases and deaths from esophageal cancer in China accounts for approximately $55 \%$ of all worldwide cases (2). Primary small cell carcinoma of the esophagus (PSCCE) is rare and aggressive. It is a highly metastatic malignancy with a poor prognosis and accounts

$\wedge$ ORCID: 0000-0002-7482-7022. 
for $0.4-2.8 \%$ of all esophageal tumors (3). Treatments, such as surgery, radiotherapy, and chemotherapy, have been performed alone or in combination to improve the survival of patients with PSCCE (4). However, an optimal therapeutic modality for PSCCE has not yet been established. Patients with PSCCE usually have a high risk of metastasis. A lack of effective treatment for PSCCE further exacerbates the global burden of this life-threatening disease.

Extensive-disease (ED) stage and limited-disease (LD) stage PSCCE are defined as primary small cell carcinoma of the esophageal with or without distant metastasis, respectively. To date, there are no standard treatments for ED- and LD-stage PSCCE. Esophagectomy and radiotherapy are widely considered as local treatments for LD-stage PSCCE patients in China $(5,6)$. Chemotherapy has been recommended as systemic therapy for ED patients. Currently, the effectiveness of various therapies on the longterm survival of PSCCE patients remains unclear, especially for patients at different lymph node stages. Thus, suitable therapeutic methods must be identified to improve the poor survival rate of PSCCE patients. In this retrospective study, we analyzed the clinical characteristics of PSCCE patients who received treatment at our center and further identified reliable prognostic factors, which can serve as valuable references in clinical practice to treat patients with PSCCE and improve their quality of life.

We present the following article in accordance with the STROBE reporting checklist (available at http://dx.doi. org/10.21037/jtd-20-3334).

\section{Methods}

\section{Patients data}

By reviewing electronic medical records, we retrospectively evaluated 113 consecutive patients with PSCCE who underwent treatment at the Sun Yat-sen University Cancer Centre between 2003 and 2016. The study was conducted in accordance with the Declaration of Helsinki (as revised in 2013). The study was approved by Research Ethics Committee of the Sun Yat-Sen University Cancer Center (NO.: GZR2018-120) and individual consent for this retrospective analysis was waived. The survival data were extracted and updated in June 2020.

\section{Eligibility and exclusion criteria}

To be selected to participate in this study, patients had to meet the following eligibility criteria: (I) have esophageal small cell carcinoma as histopathologically proven by an endoscopic biopsy or surgery; and (II) have the complete treatment and follow-up records. Patients were excluded from the study if their medical history stated they had suffered from other malignant tumors.

\section{Preoperative auxiliary examination}

The routine workup included barium esophagography, endoscopic biopsy, brain imaging, and contrast-enhanced computed tomography (CT) scans of the neck, chest, and upper abdomen. A positron emission tomography (PET) scan is recommended to exclude distant metastasis. Endoscopic ultrasonography (EUS) evaluates the extent of the lesion and the condition of regional lymph nodes. Immunohistochemical (IHC) staining was performed to determine the status of common neuroendocrine markers.

\section{Staging}

All patients were staged according to the 8th edition of the tumor $(\mathrm{T})$, nodes $(\mathrm{N})$, and metastases $(\mathrm{M})(\mathrm{TNM})$ staging system of the American Joint Committee on Cancer (AJCC) (7), and the staging system of the Veterans' Administration Lung Study Group (VALSG) (8). The VALSG system is composed of the LD-stage and EDstage. LD-stage PSCCE is characterized by limited tumoral involvement to the bilateral mediastinum (with or without local extension) and no distant extra-thoracic metastatic disease. Bilateral hilar, abdominal trunk, bilateral cervical paraoesophageal lymph nodes are considered as esophageal regional lymph nodes. However, regardless of the primary tumor location, bilateral supraclavicular lymph nodes are defined as distant metastases. All other cases, including features, such as malignant pleural and pericardial effusions, are classified as ED-stage PSCCE.

\section{Demographic and clinical data}

The clinical data of the PSCCE patients, including baseline characteristics, laboratory examinations, tumor features, and 
treatments, were extracted from medical records. According to various clinical data, patients were divided into different groups, and overall survival (OS) was analyzed.

\section{Hematology test}

Blood samples for a routine laboratory examination were collected within 3 days of the first treatment to avoid any effects related to surgery/radiotherapy or drug therapy. Assay indexes, including neutrophil, lymphocyte, platelet, and monocyte counts, were obtained from the complete blood count. We chose 3 major laboratory markers as prognostic and predictive factors, including the lymphocyteto-monocyte ratio (LMR), neutrophil-to-lymphocyte ratio (NLR), and platelet-to-lymphocyte ratio (PLR) $(9,10)$. The optimal cutoff values of these markers were calculated based on receiver operating characteristic (ROC) curves.

\section{Statistical analysis}

OS was selected as the primary endpoint and calculated from the first treatment date to the date of death or the last follow-up appointment. Descriptive analyses of patient characteristics, clinical features, and outcomes were conducted. Survival analysis was performed using the Kaplan-Meier method. Any prognostic factor that was significant in the univariate analysis was selected and included in the multivariate analysis, which was performed using a Cox regression model. After the multivariate Cox analysis, factors with significant differences could be defined as independent prognostic factors. A P value of $<0.05$ indicated a significant difference. All statistical analyses were performed using the SPSS software version 22.0 for Windows (IBM Corp, Armonk, NY, USA).

\section{Results}

\section{Patients and clinical baseline characteristics}

The demographic and clinicopathological characteristics of the 113 patients are set out in Table 1. Eighty-seven men and 26 women were included in the analysis with a median age of 58.8 years (ranging from 35 to 76 years). Tumors were mostly located in the middle $(48.7 \%)$ and lower thoracic $(38.9 \%)$ esophagus segments. The lengths of the lesions ranged from 1.2 to $12.0 \mathrm{~cm}$.

In our study, treatments for PSCCE include surgery alone, chemotherapy, surgery and postoperative chemotherapy, palliative radiotherapy, chemoradiotherapy, and trimodality therapy, including surgery and postoperative chemoradiotherapy. Figure 1 shows a treatment flowchart, which was made by summarizing therapeutic experiences at our center. Concerning the therapeutic regimen, 49 patients were treated with surgery. About surgical procedures, 27 patients $(55.1 \%)$ underwent Sweet surgery, 19 patients (38.8\%) underwent McKeown surgery, and 3 patients (6.1\%) underwent Ivor-Lewis surgery (see Table 2). A total of 990 lymph nodes were resected during surgery, with an average of 20.2 lymph nodes per patient. Among the dissected lymph nodes, 148 (14.9\%) lymph nodes were pathologically confirmed as small cell carcinoma metastases. When faced with chemotherapy options, most patients were treated with a regimen of etoposide plus cisplatin (EP, $67.5 \%$ ) or paclitaxel plus cisplatin (TP, $20.5 \%)$. Intensitymodulated radiation therapy (IMRT) was the first choice of radiotherapy for PSCCE.

\section{Pathological diagnosis by preoperative biopsy and postoperative pathology and IHC positive staining}

Forty-nine patients underwent postoperative pathology examinations. The histopathological test results revealed that a single pathological type, PSCCE, was observed in 44 patients $(89.8 \%)$ with esophageal neoplasms. The esophagus lesions of the remaining 5 patients (10.2\%) showed mixed histology of PSCCE and squamous cell carcinoma (SCC) or adenocarcinoma. Compared with the postoperative pathology results, the preoperative diagnosis's accuracy rate by endoscopic biopsy was $42.9 \%$ (21/49). An endoscopic biopsy analysis showed that 12 patients (24.5\%) suffered from undifferentiated or poorly differentiated carcinomas, and 16 patients $(32.6 \%)$ were misdiagnosed with SCC or adenocarcinoma before surgery.

Postoperative IHC staining was usually performed to determine the common neuroendocrine markers of PSCCE for further diagnosis. Ten common immunological indicators of PSCCE were selected, and the positive expressions of the tissue samples of our PSCCE patients were identified from highest to lowest positivity (see Table 3). CD56 and low molecular weight cytokeratin (CK-L) were identified as $100 \%$ positive in all tissue samples. The median Ki-67 (a nuclear protein associated with cellular proliferation) labeling index was $80 \%$ (ranging from $60 \%$ to $90 \%$ ). 
Table 1 Patient information and clinical characteristics

\begin{tabular}{|c|c|c|}
\hline Variable & $n$ & $\%$ \\
\hline \multicolumn{3}{|l|}{ Sex } \\
\hline Male & 87 & 77.0 \\
\hline Female & 26 & 23.0 \\
\hline \multicolumn{3}{|l|}{ Age } \\
\hline$<65$ years & 82 & 72.6 \\
\hline$\geq 65$ years & 31 & 27.4 \\
\hline \multicolumn{3}{|l|}{ Location } \\
\hline Upper thoracic & 12 & 10.6 \\
\hline Middle thoracic & 55 & 48.7 \\
\hline Lower thoracic & 44 & 38.9 \\
\hline Multiple & 2 & 1.8 \\
\hline \multicolumn{3}{|l|}{ Tumor depth } \\
\hline T1 & 9 & 8.0 \\
\hline T 2 & 35 & 31.0 \\
\hline T 3 & 46 & 40.7 \\
\hline T 4 & 7 & 6.2 \\
\hline NA & 16 & 14.1 \\
\hline \multicolumn{3}{|l|}{ Lymph node } \\
\hline No & 24 & 21.2 \\
\hline $\mathrm{N} 1$ & 33 & 29.2 \\
\hline N 2 & 26 & 23.0 \\
\hline N 3 & 19 & 16.8 \\
\hline NA & 11 & 9.8 \\
\hline \multicolumn{3}{|c|}{ Distance metastasis } \\
\hline M 0 & 84 & 74.3 \\
\hline M 1 & 29 & 25.7 \\
\hline \multicolumn{3}{|l|}{ TNM } \\
\hline Stage I & 3 & 2.7 \\
\hline Stage II & 26 & 23.0 \\
\hline Stage III & 37 & 32.7 \\
\hline Stage IV & 47 & 41.6 \\
\hline \multicolumn{3}{|l|}{ Treatment } \\
\hline Surgery & 26 & 23.0 \\
\hline Chemotherapy & 20 & 17.7 \\
\hline
\end{tabular}

Table 1 (continued)
Table 1 (continued)

\begin{tabular}{lcc}
\hline Variable & $\mathrm{n}$ & $\%$ \\
\hline Surgery + chemotherapy & 19 & 16.8 \\
Radiotherapy & 2 & 1.8 \\
chemoradiotherapy & 42 & 37.2 \\
$\begin{array}{l}\text { Surgery + } \\
\text { chemoradiotherapy }\end{array}$ & 4 & 3.5 \\
Radiotherapy modality & & \\
3D-CRT & 9 & 20.0 \\
IMRT & 26 & 57.8 \\
NA & 10 & 22.2 \\
Chemotherapy regimen & & \\
EP & 56 & 67.5 \\
TP & 17 & 20.5 \\
Other & 10 & 12.0 \\
\hline
\end{tabular}

T, tumor; $N$, regional lymph node; $M$, metastasis; NA, not available; 3D-CRT, 3-dimensional conformal radiation therapy; IMRT, intensity modulated radiation therapy; EP, etoposide and cisplatin chemotherapy; TP, paclitaxel and cisplatin chemotherapy.

\section{OS analysis}

By June 30, 2020, the median follow-up time for the 113 patients was 16.3 months (ranging from 2.33 to 198.5 months). Among the 113 patients, 75 patients (66.4\%) died of tumor recurrence or metastases, 26 (23.0\%) were still alive, and 12 patients (10.6\%) were lost to followup. Based on the data, the mean survival time (MST) of the whole group was 22.9 months. The 6-, 12-, 24-, and 36 -month OS rates were $64 \%, 45 \%, 22 \%$, and $12 \%$, respectively (see Figure 2).

\section{The univariate analysis revealed that lymph node stage, VALSG staging, treatment modalities, and the inflammation biomarker LMR were significantly correlated with the OS of PSCCE patients}

To evaluate the correlations between patient survival and clinicopathological features, several elements, such as gender, age, tumor location, tumor depth, regional lymph node stage, VALSG staging, treatment modalities, and inflammation biomarkers, were chosen and analyzed one 


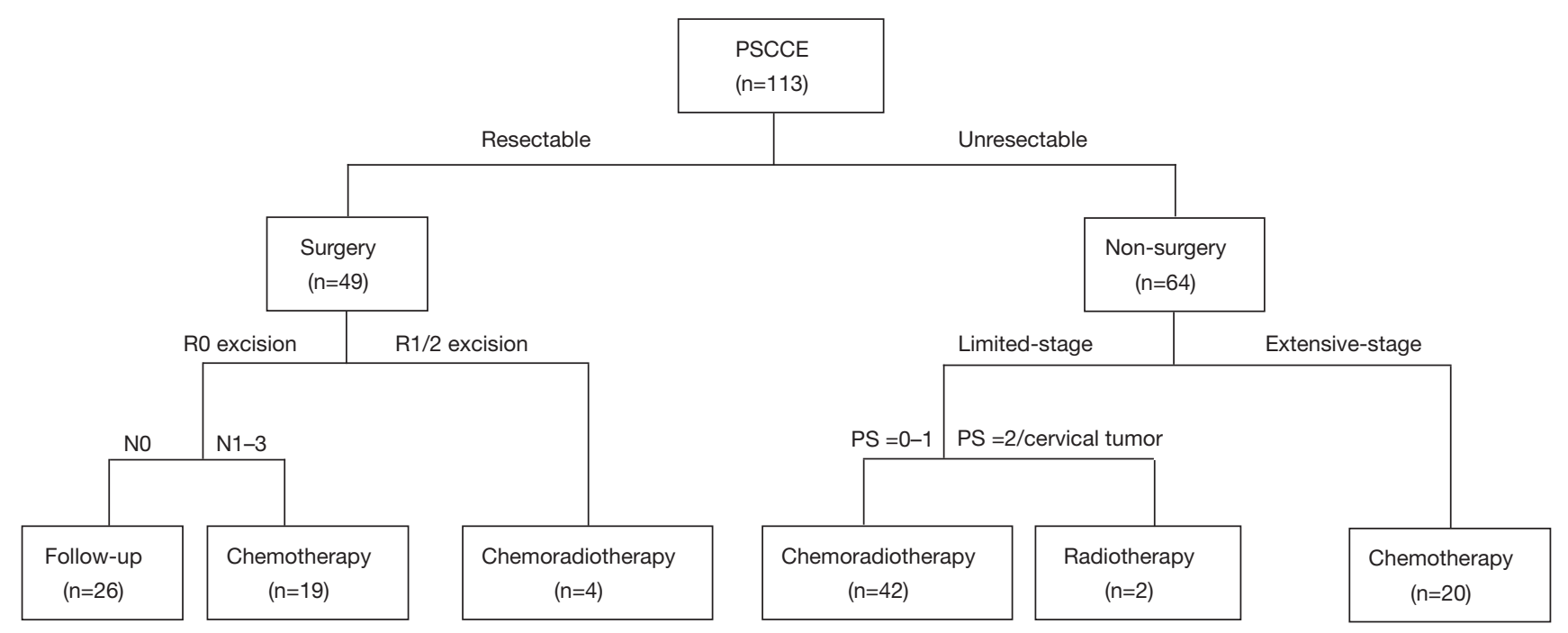

Figure 1 Treatment flowchart for primary small cell carcinoma of the esophagus.

Table 2 Information and tumor characteristics of 49 patients undergoing surgery

\begin{tabular}{|c|c|c|}
\hline Variable & Num. & Percentage \% \\
\hline \multicolumn{3}{|l|}{ Treatment } \\
\hline Surgery & 26 & 53.1 \\
\hline Surgery + adjuvant therapies & 18 & 36.7 \\
\hline $\begin{array}{l}\text { Neoadjuvant therapy + surgery } \\
\text { + adjuvant therapies }\end{array}$ & 5 & 10.2 \\
\hline \multicolumn{3}{|l|}{ Surgical procedure } \\
\hline Sweet & 27 & 55.1 \\
\hline Ivor-Lewis & 3 & 6.1 \\
\hline Mckeown & 19 & 38.8 \\
\hline \multicolumn{3}{|l|}{ Postoperative pathology } \\
\hline Pure SCCE & 44 & 89.8 \\
\hline SCCE + SCC/adenocarcinoma & 5 & 10.2 \\
\hline \multicolumn{3}{|l|}{ Endoscopic biopsy pathology } \\
\hline Small cell carcinoma & 21 & 42.9 \\
\hline Poorly differentiated carcinoma & 12 & 24.5 \\
\hline $\begin{array}{l}\text { Inaccurate pathological } \\
\text { diagnosis }\end{array}$ & 16 & 32.6 \\
\hline \multicolumn{3}{|l|}{ Anastomotic leak } \\
\hline Yes & 7 & 14.3 \\
\hline No & 42 & 85.7 \\
\hline
\end{tabular}

Num, number; SCCE, small cell carcinoma of esophagus; SCC, squamous cell carcinoma. by one. The $\mathrm{T}$ and $\mathrm{N}$ stages of PSCCE were categorized as T1-2 and T3-4, N0-1 and N2-3, respectively. The treatment modalities were classified as a single (surgery or chemotherapy alone) and combined treatment (surgery combined with postoperative chemotherapy). As Table 4 shows, lymph node stage [hazard ratio (HR) $=0.555 ; 95 \%$ confidence interval (CI): 0.356-0.867; $\mathrm{P}=0.009]$, VALSG staging $(\mathrm{HR}=0.618 ; 95 \% \mathrm{CI}: 0.391-0.978 ; \mathrm{P}=0.038)$, treatment method ( $\mathrm{HR}=1.743 ; 95 \% \mathrm{CI}: 1.159-2.642$; $\mathrm{P}=0.046$ ), and the inflammation biomarker LMR (HR $=0.332 ; 95 \%$ CI: $0.140-0.788 ; \mathrm{P}=0.009$ ) were significantly associated with the OS of PSCCE patients according to the univariate analysis.

As Figure $3 A$ shows, patients at $\mathrm{N} 0-1$ stage PSCCE had longer OS rates than those at $\mathrm{N} 2-3$ stage $(\mathrm{P}=0.009)$. Stratified analysis was conducted to investigate further the effect of the various lymph node stages on the MST of PSCCE patients. The stratified analysis showed that the MST at stage N0 was longer than that at stage N1, N2, and N3 (22.5 versus 22.2 versus 10.7 versus 9.7 months, respectively; $\mathrm{P}<0.001$, see Figure $3 B$ ). Concerning VALSG staging, the MST of patients in the LD stage was markedly longer than that of patients in the ED stage (17.5 versus 12.6 months, $\mathrm{P}=0.038$, Figure 4).

Also, the MST of surgery/chemotherapy alone was shorter than that of surgery combined with postoperative chemotherapy (10.4 versus 17.8 months; $\mathrm{P}=0.001$, Figure $5 A$ ). Stratified analysis was conducted to investigate the effect of treatment modalities on the MST of PSCCE 
Table 3 Immunohistochemical characteristics of PSCCE patients undergoing surgery

\begin{tabular}{lcccccccccc}
\hline IHC & CD56 & CK-L & NSE & CK & EMA & Syn & TTF-1 & CgA & CK-56 & P63 \\
\hline Num. & $35 / 35$ & $9 / 9$ & $35 / 39$ & $31 / 35$ & $8 / 10$ & $38 / 42$ & $16 / 25$ & $23 / 43$ & $9 / 26$ & $10 / 35$ \\
Per. (\%) & 100.0 & 100.0 & 89.7 & 88.6 & 80.0 & 79.2 & 64.0 & 53.5 & 34.6 & 28.6 \\
\hline
\end{tabular}

PSCCE, primary small cell carcinoma of esophagus; IHC, immunohistochemical staining; Num, number; Per, percent; CD56, neural cell adhesion molecule 1; CK-L, low molecular weight cytokeratin; NSE, neuron-specific enolase; CK, cytokeratin; EMA, epithelial membrane antigen; Syn, synaptophysin; TTF-1, thyroid transcription factor 1; CgA, chromogranin A; CK-56, cytokeratin 56; P63, tumor protein P63.

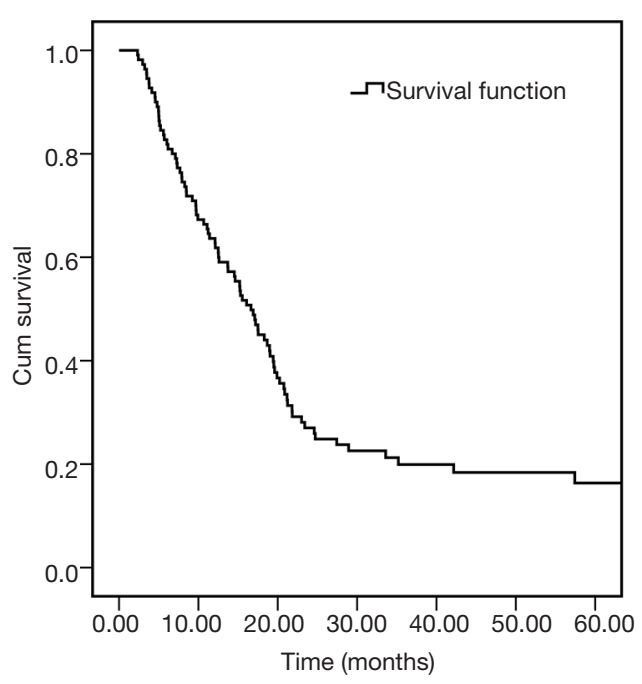

Figure 2 Kaplan-Meier survival curve of OS for 113 patients with primary small cell carcinoma of esophagus. OS, overall survival.

patients at distinct lymph node stages and VALSG staging. Based on the stratified data, the benefits of surgery combined with chemotherapy were observed in N1-3 patients (see Figure 5B), but systemic treatments did not show an obviously beneficial effect among N0 patients (see Figure S1). In relation to different chemotherapy regimens, an EP regimen prolonged the OS of VALSG ED-stage patients more than a TP regimen (see Figure 5C), while LD-stage patients rarely benefited more from an EP regime.

Concerning the inflammation biomarkers, the optimal cutoff points for the LMR, NLR, and PLR were determined to be 5.13, 4.6, and 143.19, respectively, based on a ROC analysis. According to the expression levels of each biomarker, the patients were divided into the following groups: (I) the low LMR group (<5.13) versus the high LMR group ( $\geq 5.13)$; (II) the low NLR group $(<4.6)$ versus the high NLR group $(\geq 4.6)$; and (III) the low PLR group $(<143.19)$ versus the high PLR group $(\geq 143.19)$. The survival analysis indicated that patients in the high LMR group had significantly better OS rates than those in the low LMR group (HR $=0.332 ; 95 \%$ CI: $0.140-0.788 ; \mathrm{P}=0.009$ ) (see Figure 6). No significant difference was observed among the patients in the different NLR and PLR groups. The other clinical features, including gender, age, tumor location, and tumor depth, did not show any significant correlations with survival prognosis.

\section{The multivariate analysis indicated that stages NO- N1, VALSG LD staging, and combination therapy were favorable predictors of OS in PSCCE patients}

Multivariate survival analysis was performed using the Cox proportional hazards model to examine further the favorable prognostic factors of OS in PSCCE patients. Consistent with the univariate analysis, the multivariate analysis suggested that the lymph node stage, the VALSG staging system, and the treatment method were independent predictors of OS in the whole cohort (see Table 5). Patients at lymph node stage N0-N1 had a better prognosis than those at stages N2-N3. Additionally, PSCCE patients with neoplasms at the ED-stage had a poorer prognosis than those with neoplasms at the LD-stage.

\section{Recurrences and metastases}

After local or systemic therapy, 35 patients $(31.0 \%)$ in the cohort experienced recurrences or metastases, including liver (15 patients), bone ( 8 patients), brain (6 patients), and supraclavicular lymph nodes (4 patients) metastases. The interval time between the first treatment and recurrence ranged from 30 days to 20 months.

\section{Discussion}

McKeown first described the epidemiological manifestations of PSCCE, which is characterized by early dissemination 
Table 4 Univariate overall survival analysis by Cox's proportional hazards model

\begin{tabular}{|c|c|c|c|}
\hline Variable & $\mathrm{HR}$ & $95 \% \mathrm{Cl}$ & $P$ value \\
\hline \multicolumn{4}{|l|}{ Gender } \\
\hline Male versus female & 0.915 & $0.718-1.167$ & 0.475 \\
\hline \multicolumn{4}{|l|}{ Age } \\
\hline$<65$ versus $\geq 65$ years & 1.075 & $0.667-1.734$ & 0.766 \\
\hline $\begin{array}{l}\text { Middle/lower versus cervical/upper } \\
\text { esophagus }\end{array}$ & 1.471 & $0.735-2.946$ & 0.276 \\
\hline \multicolumn{4}{|l|}{$\mathrm{T}$} \\
\hline T1-2 versus T3-4 & 1.145 & $0.721-1.820$ & 0.566 \\
\hline \multicolumn{4}{|l|}{$\mathrm{N}$} \\
\hline LD versus ED & 0.618 & $0.391-0.978$ & 0.038 \\
\hline \multicolumn{4}{|l|}{ Treatment } \\
\hline$S / C$ versus $S+C$ & 1.743 & $1.159-2.642$ & 0.046 \\
\hline \multicolumn{4}{|l|}{ LMR } \\
\hline Low versus high & 0.332 & $0.140-0.788$ & 0.009 \\
\hline \multicolumn{4}{|l|}{ NLR } \\
\hline Low versus high & 0.776 & $0.409-1.471$ & 0.437 \\
\hline
\end{tabular}

HR, hazard ratio; Cl, confidence interval; T, tumor; N, regional lymph node; VALSG, Veterans' Administration Lung Study Group; LD, limited-stage disease; ED, extensive-stage disease; S, surgery; R, radiotherapy; C, chemotherapy; LMR, lymphocyte-to-monocyte ratio; NLR, neutrophil-to-lymphocyte ratio; PLR, platelet-to-lymphocyte ratio.

and a poor prognosis (11). PSCCE mostly occurs in older men and is located in the middle and lower thoracic esophagus (12). It is difficult to distinguish PSCCE from SCC or adenocarcinoma by macroscopic tests or imaging examinations. Additionally, the coexistence of PSCCE and SCC or adenocarcinoma in the same lesion also increases diagnosis difficulty. In the present study, carcinomas with mixed histology were observed in $10.2 \%$ of patients, consistent with the incidence of $9.4 \%$ to $38.4 \%$ cited in previous reports (12).

With the diagnosis of PSCCE, a biopsy through esophagoscopy and paraffin section tissue removal during surgery were used the most frequently. Additionally, in conjunction with clinical examinations and cytomorphology, IHC has become an indispensable tool in the postoperative diagnosis of PSCCE (13). Histologically, PSCCE is characterized by a neuroendocrine-like architectural pattern, which is similar to small cell lung cancer (SCLC). It is usually difficult to distinguish PSCCE from other esophageal poorly differentiated cells. The pathological diagnosis depends mainly on IHC staining positivity for several common neuroendocrine factors (14).

The accurate rate of preoperative diagnosis in our study was only $42.9 \%$. This low rate may have been caused by limitations related to endoscopic biopsy or an absence of IHC staining. About the biopsy samples, $24.5 \%$ samples were diagnosed as poorly differentiated carcinoma, and $32.6 \%$ were misdiagnosed as non-small cell carcinoma. With the rate of misdiagnosis of PSCCE, it should be noted that many patients with PSCCE receive ineffective 


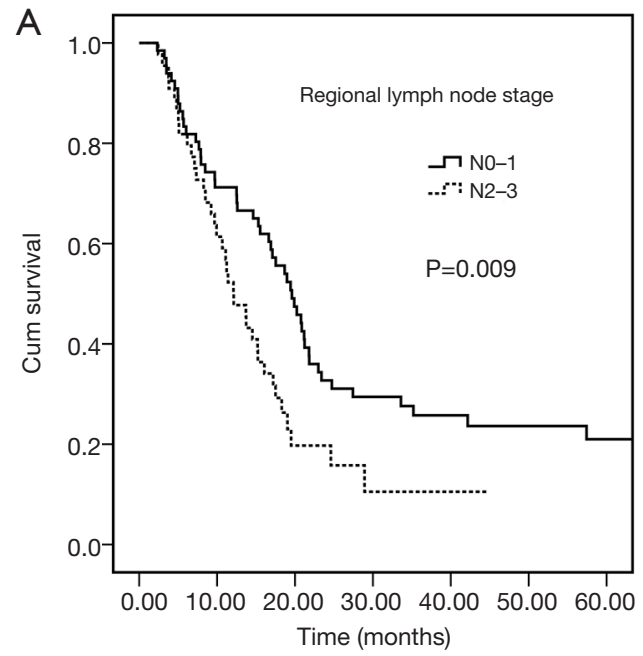

B

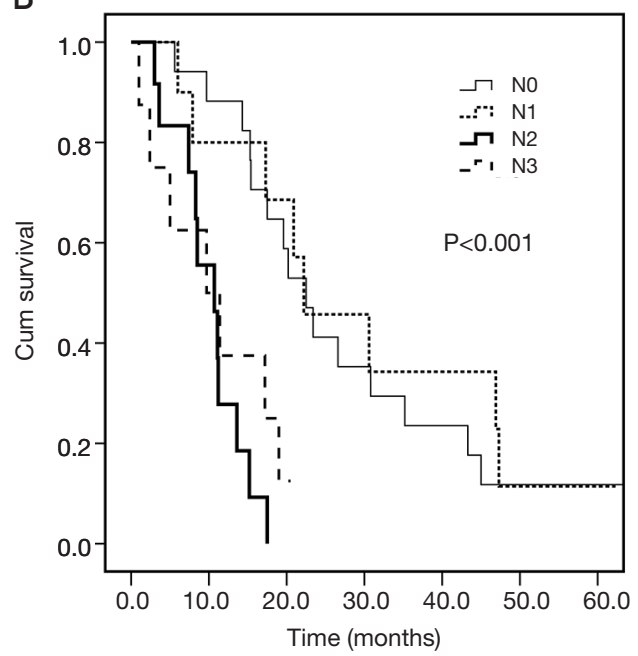

Figure 3 Kaplan-Meier survival curves of OS for patients at different $\mathrm{N}$ stages. (A) N0-1 versus N2-3. (B) N0 versus N1, N2, and $\mathrm{N} 3$, respectively. OS, overall survival.

treatments, such as non-sensitive chemotherapy regimens, as PSCCE cannot be easily identified and precisely diagnosed, which contributes to an unfavorable prognosis. Thus, it is necessary to improve the diagnosis accuracy of PSCCE through multidisciplinary collaborations. Endoscopy physicians should extract tumor samples with strict adherence to standard protocols. Pathologists need to perform IHC staining after microscopy despite the timeconsuming nature of this task and the high associated costs. Accurate diagnoses could provide patients and physicians with more valuable information, which would further help them to make decisions and improve their prognoses.

Regional lymph node metastasis in PSCCE patients

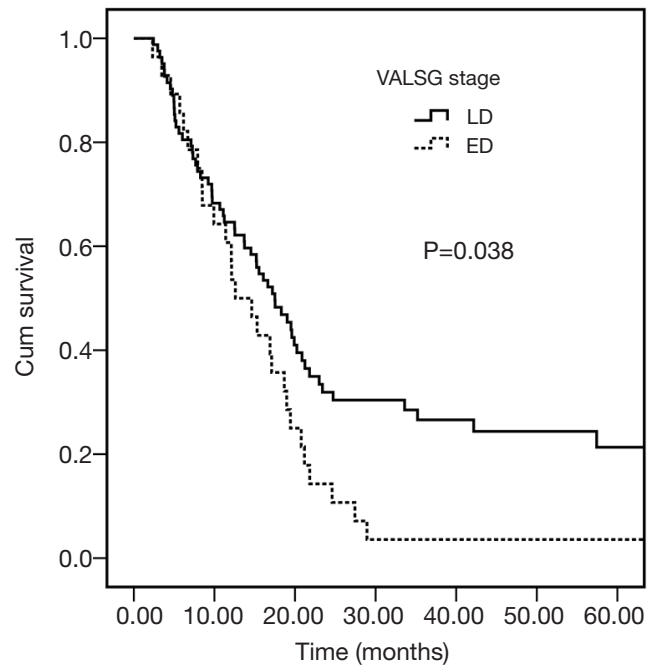

Figure 4 Kaplan-Meier survival curve of OS for patients at different VALSG stages (LD versus ED). OS, overall survival; VALSG, Veterans' Administration Lung Study Group; LD, limited disease; ED, extensive disease.

develops early due to the characteristics of rapid progression and aggressive invasion (11). The metastasis rate was $76.5 \%$ in our study, which was consistent with the rate of $79.0 \%$ reported in a previous study (6). This further emphasizes the importance of the accurate and early diagnosis of PSCCE. To assess PSCCE staging accurately, we recommend that all PSCCE patients undergo PET scans and EUS examinations. Combined with contrast-enhanced computed tomography (CT), EUS examinations provide detailed information about the extent of a lesion, and the condition of regional lymph node metastasis. A PET scan is an essential preoperative auxiliary examination that can be used to exclude distant metastasis.

Another significant characteristic of PSCCE was the unsatisfactory prognosis. Thus, we analyzed the potential prognostic factors of PSCCE and explored optimal treatment strategies. Our research revealed that the regional lymph node stage was an independent prognostic factor for PSCCE patients. Patients with limitedly metastatic lymph nodes achieved a favorable prognosis. Similar to the results of previous studies (15), the present study showed that the MST of patients at stage N0 was longer than that of patients at stages N1, N2, or N3. Sun et al. proposed that patients who develop only a few lymph node metastases have longer OS than those with marked lymph node metastases (16). Zhang and Situ et al. also noted the vital role of lymph node 

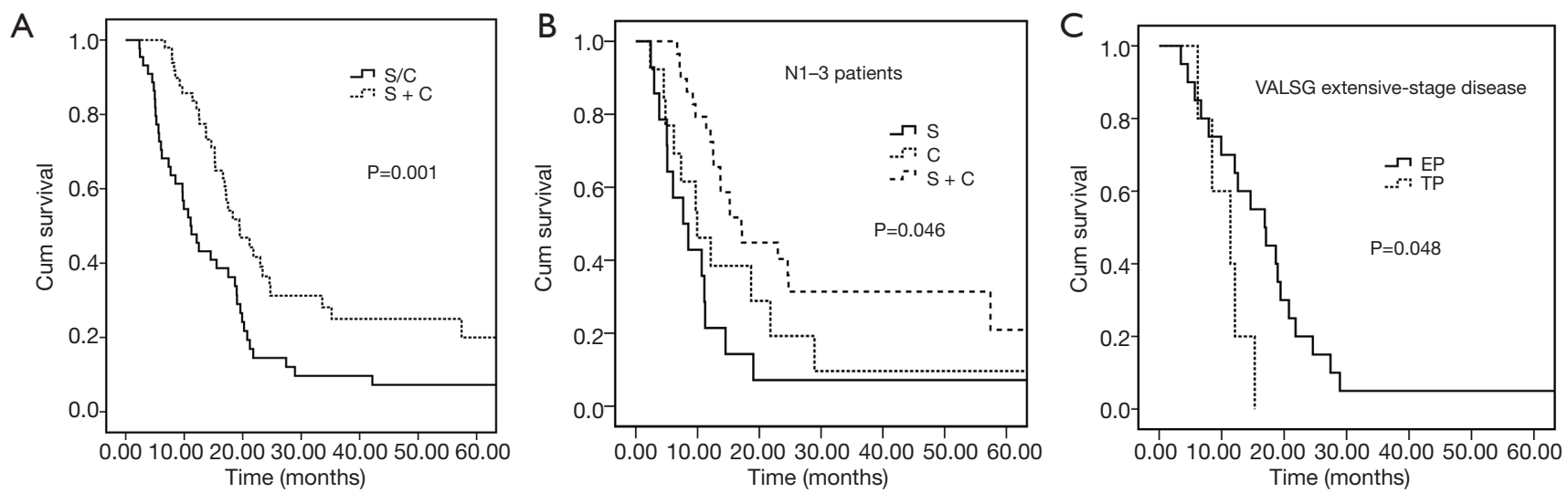

Figure 5 Kaplan-Meier survival curves of OS for patients treated with different treatments. (A) Kaplan-Meier survival curve of OS for patients treated with S/C or S + C. (B) Kaplan-Meier survival curve of OS for N1-N3 patients treated with S/C or S + C. (C) KaplanMeier survival curve of OS for extensive-disease stage patients treated with an EP versus a TP regime. OS, overall survival; S, surgery; C, chemotherapy; EP, etoposide plus cisplatin; TP, paclitaxel plus cisplatin.

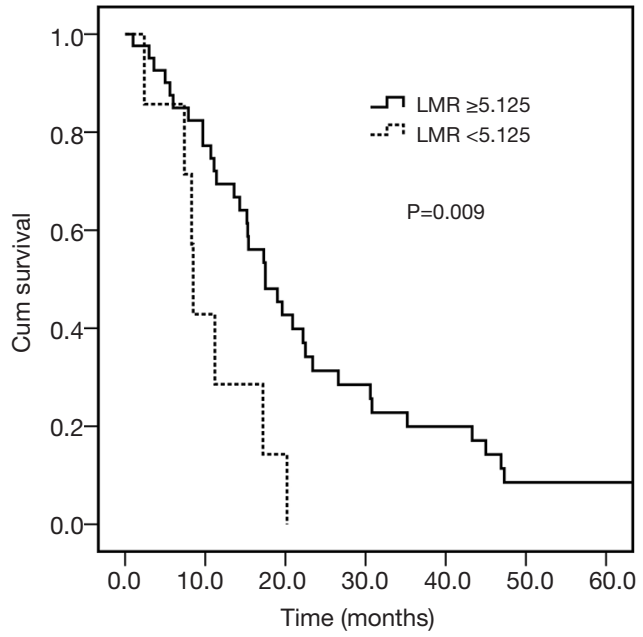

Figure 6 Kaplan-Meier survival curve of OS for patients in the high LMR group $(\geq 5.125)$ versus the low LMR group $(<5.125)$. OS, overall survival; LMR, lymphocyte-to-monocyte ratio.

involvement as an independent prognostic factor for $\mathrm{LD}$ stage PSCCE patients $(17,18)$.

There is overwhelming evidence that a comprehensive understanding of esophageal tumors' development requires further research of tumor microenvironments, which can trigger chronic inflammation and promote the proliferation of tumor cells (19). Systemic inflammation is often involved in the process of tumor formation and could promote tumor invasion (20). Cancer-related inflammation biomarkers, such as the LMR, NLR, and PLR, are expected to predict survival prognosis (21). The LMR is a comprehensive inflammatory indicator that is associated with the prognosis of several malignancies, such as lung cancer, colorectal cancer, and esophageal SCC $(22,23)$. In our study, patients in the low LMR group had significantly poorer OS than those in the high LMR group. Thus, LMR appears to be a supplemental factor in the prognostic prediction of PSCCE.

Different modalities were chosen depending on the characteristics of the tumors and the physical condition of patients. To date, no consensus has been reached concerning a standard therapy modality. Notably, some oncologists recommended that the treatment protocol for PSCCE resemble that of SCLC's treatment protocol. In their opinion, PSCCE is a systemic disease, and patients should undergo chemotherapy as SCLC patients do (15). Conversely, other oncologists believe that surgical interventions should serve as the primary treatment for PSCCE (24), that surgery should be performed in patients at the LD-stage, and that the treatment principle for PSCCE should be similar to that for esophageal squamous carcinoma (25). In the present study, we divided the modalities of PSCCE into surgery and non-surgery treatment according to tumor resectability.

In China, esophagectomy is the most popular local therapy and is performed in $52 \%$ to $85 \%$ of PSCCE cases (25). Surgery is highly recommended for patients with good performance status and resectable tumors. To be eligible for surgery, PSCCE patients must meet the following criteria: (I) be at a TNM stage of I, II, or III; 
Table 5 Multivariate analysis for prognostic factors of overall survival

\begin{tabular}{|c|c|c|c|}
\hline Variable & $\mathrm{RR}$ & $95 \% \mathrm{Cl}$ & $P$ value \\
\hline N0-1 versus N2-3 & 0.496 & $0.317-0.778$ & 0.022 \\
\hline \multicolumn{4}{|l|}{ VALSG } \\
\hline LD versus ED & 0.456 & $0.279-0.746$ & 0.040 \\
\hline$S / C$ versus $S+C$ & 2.639 & $1.492-4.331$ & 0.047 \\
\hline \multicolumn{4}{|l|}{ LMR } \\
\hline Low versus high & 0.605 & $0.246-1.489$ & 0.274 \\
\hline
\end{tabular}

RR, relative risk; Cl, confidence interval; N, regional lymph node; VALSG, Veterans' Administration Lung Study Group; LD, limited-stage disease; ED, extensive-stage disease; S, surgery; C, chemotherapy; LMR, lymphocyte-to-monocyte ratio.

(II) a tumor stage of 1, 2, or 3 (T1, T2, or T3); (III) show no nodal involvement (N0), or the presence of tumor(s) in no more than 6 lymph nodes (N1-2); (IV) and show no metastases (M0); (V) have esophageal cancer in the middle or lower third of the esophagus; (VI) have a World Health Organization performance-status score of 0 or 1 (on a 5 -point scale) (26). Detailed postoperative pathology examinations provide an opportunity to eliminate potential misdiagnoses of preoperative pathology results provided by bioscopies. In the present study, 7 patients experienced anastomotic leakage after surgery; however, the incidental complications appeared not to affect their OS. Consistent with our findings, $\mathrm{Xu}$ et al. indicated that patients treated with surgery had a better survival rate than those who received non-surgical therapies (27). Also, Zou (28) and Chen (1) et al. showed that surgery had a significant role in improving PSCCE patients' survival.

Long-term follow-up examinations may be acceptable for patients without lymph node metastasis after an R0 excision. In the present study, we explored the correlation between treatment methods and the prognosis of PSCCE and found that N1-3-stage (but not N0-stage) patients who were treated with surgery combined with chemotherapy had a better prognosis than those who underwent surgery or chemotherapy alone. Thus, combined with our data, we recommended that N1-N3-stage PSCCE patients receive adjuvant therapy after surgery. Due to chemotherapy's potential side effects, we do not recommend overenthusiastic chemotherapy for early-stage PSCCE patients. Concerning patients who received an $\mathrm{R} 1 / 2$ excision, postoperative chemoradiotherapy is essential to eliminate the residual lesion (29). With combination therapy, patients' symptoms, such as dysphagia, were relieved by surgery, and the OS rate of patients with lymph node metastasis was effectively prolonged by chemotherapy. As PSCCE can possess aggressive characteristics, which manifest in early regional lymph node metastasis, many patients are diagnosed with advanced tumors after surgery and obtain very few benefits from monotherapy treatments when their tumor(s) spread to other locations.

For patients with tumor metastasis, chemotherapy is the key treatment to destroy metastatic tumor cells and control disease progression. PSCCE was first treated with chemotherapy by Kelsen (30). Chemotherapy could improve both LD- and ED-stage patients' survival as a part of multimodality therapy (31). Raja reviewed 148 articles, analyzed the survival of 577 PSCCE patients, and found that chemotherapy is the optimal strategy for PSCCE (32).

About chemotherapy for SCC, a union regimen in which cisplatin (CDDP) is involved should be considered first (31). However, the detailed principle of PSCCE chemotherapy has not yet been clearly illustrated. In the present study, most patients still chose a regimen of etoposide plus cisplatin (EP), followed by paclitaxel plus cisplatin (TP). In our cohort, an EP regime resulted in better survival than a TP regime in VALSG ED-stage patients; However, the same advantages were not found in VALSG LD-stage patients. With the OS of PSCCE patients, we found that the MST for the entire group of PSCCE patients was 16.3 months, and the 3 -year OS rate was only $12 \%$, which was lower than the 3 -year OS rate of $30 \%$ reported by Jeene et al. in 2019 (6). After conducting a detailed analysis and comparison, we observed that the percentage of patients treated with chemotherapy in our cohort $(77.0 \%)$ was 
significantly lower than that in Jeene's cohort (92\%), which may be why the OS rate is unsatisfactory compared to that of other studies. Most patients in our study died of liver, bone, or brain metastases after treatment, which further confirmed the poor prognosis of PSCCE.

Radiotherapy is an effective treatment for PSCCE, especially in patients with cervical esophageal cancer, which is sensitive to radiotherapy but not suitable for surgery (33). Radiotherapy is often used as an adjuvant therapy pre- or post-surgery (34). The treatment of radiotherapy alone is only suitable for patients who cannot afford surgery or concurrent chemoradiotherapy, especially those with poor performance status.

In recent years, more oncologists at our center have chosen neoadjuvant chemoradiotherapy (CRT) followed by surgery to treat PSCCE (35). However, the prognosis of patients with neoadjuvant CRT is not clear yet. For patients with a T4 tumor, we recommend a neoadjuvant CRT to shrink the tumor before surgery. With the development of immunotherapy, immunotherapy combined with chemotherapy can treat PSCCE patients, especially those with unresectable tumors, recurrence, or distal metastasis. Many ongoing clinical trials are being conducted at our center, and the results could provide useful insights into PSCCE treatments in the future. However, immunotherapy's side effects, including myocarditis, pneumonitis, hepatitis, and encephalitis, need to be considered (35).

This study's limitations include its small sample size and the data gathered from a single institution. Due to the limited number of patients, we could not fully evaluate the differences between the effects of postoperative chemotherapy and neoadjuvant chemotherapy on the survival outcome. A large, prospective, multicentre, and randomized controlled trial needs to be conducted to confirm this study's results.

\section{Conclusions}

PSCCE is a rare type of esophageal tumor with high malignancy, early metastasis, and poor prognosis. Our study indicated that lymph node stage, VALSG staging, treatment modalities, and the inflammatory biomarker LMR were related to PSCCE patients' survival. An early lymph node stage, no distant metastasis, and multimodality treatment were independently favorable prognostic factors of PSCCE. Different modalities were chosen depending on the characteristics of tumors and the physical condition of patients. We recommend postoperative chemotherapy combined with surgery as the primary treatment for LDstage PSCCE patients at N1-3 stages.

\section{Acknowledgments}

The language of the paper was edited by AME Editing Service Editors: L. Huleatt and J. Chapnick.

Funding: This work was supported by the "National Natural Science Foundation of China (81871986)".

\section{Footnote}

Reporting Checklist: The authors have completed the STROBE reporting checklist. Available at http://dx.doi. org/10.21037/jtd-20-3334

Data Sharing Statement: Available at http://dx.doi. org/10.21037/jtd-20-3334

Peer Review File: Available at http://dx.doi.org/10.21037/jtd20-3334

Conflicts of Interest: All authors have completed the ICMJE uniform disclosure form (available at http://dx.doi. org/10.21037/jtd-20-3334). The authors have no conflicts of interest to declare.

Ethical Statement: The authors are accountable for all aspects of the work in ensuring that questions related to the accuracy or integrity of any part of the work are appropriately investigated and resolved. The study was conducted in accordance with the Declaration of Helsinki (as revised in 2013). The study was approved by Research Ethics Committee of the Sun Yat-Sen University Cancer Center (NO.: GZR2018-120) and individual consent for this retrospective analysis was waived.

Open Access Statement: This is an Open Access article distributed in accordance with the Creative Commons Attribution-NonCommercial-NoDerivs 4.0 International License (CC BY-NC-ND 4.0), which permits the noncommercial replication and distribution of the article with the strict proviso that no changes or edits are made and the original work is properly cited (including links to both the formal publication through the relevant DOI and the license). 
See: https://creativecommons.org/licenses/by-nc-nd/4.0/.

\section{References}

1. Chen WW, Wang F, Chen S, et al. Detailed analysis of prognostic factors in primary esophageal small cell carcinoma. Ann Thorac Surg 2014;97:1975-81.

2. Bray F, Ferlay J, Soerjomataram I, et al. Global cancer statistics 2018: GLOBOCAN estimates of incidence and mortality worldwide for 36 cancers in 185 countries. CA Cancer J Clin 2018;68:394-424.

3. Krishnatreya M, Kataki AC, Sharma JD, et al. Epidemiology of primary small cell carcinoma of the esophagus: a retrospective study. South Asian J Cancer 2014;3:231-2.

4. Wong AT, Shao M, Rineer J, et al. Treatment and survival outcomes of small cell carcinoma of the esophagus: an analysis of the National Cancer Data Base. Dis Esophagus 2017;30:1-5.

5. Xiao Q, Xiao H, Ouyang S, et al. Primary small cell carcinoma of the esophagus: comparison between a Chinese cohort and surveillance, epidemiology, and end results (SEER) data. Cancer Med 2019;8:1074-85.

6. Jeene PM, Geijsen ED, Muijs CT, et al. Small cell carcinoma of the esophagus: a Nationwide analysis of treatment and outcome at patient level in Locoregional disease. Am J Clin Oncol 2019;42:534-8.

7. Rice TW, Ishwaran H, Ferguson MK, et al. Cancer of the esophagus and esophagogastric junction: an 8 edition staging primer. J Thorac Dis 2017;9:E282-4.

8. Carter BW, Glisson BS, Truong MT, et al. Small cell lung carcinoma: staging, imaging, and treatment considerations. Radiographics 2014;34:1707-21.

9. Coussens LM, Werb Z. Inflammation and cancer. Nature 2002;420:860-7.

10. Balkwill F, Mantovani A. Inflammation and cancer: Back to Virchow? Lancet 2001;357:539-45.

11. McKeown F. Oat-cell carcinoma of the oesophagus. J Pathol Bacteriol 1952;64:889-91.

12. Tao H, Li F, Wang J, et al. Management of treatmentnaïve limited-stage small cell esophagus carcinoma. Saudi Med J 2015;36:297-303.

13. Duan K, Mete O. Algorithmic approach to neuroendocrine tumors in targeted biopsies: Practical applications of immunohistochemical markers. Cancer Cytopathol 2016;124:871-84.

14. Beasley MB. Immunohistochemistry of pulmonary and pleural neoplasia. Arch Pathol Lab Med 2008;132:1062-72.
15. Rice TW, Blackstone EH, Rusch VW. 7th edition of the AJCC Cancer Staging Manual: esophagus and esophagogastric junction. Ann Surg Oncol 2010;17:1721-4.

16. Sun KL, He J, Cheng GY, et al. Management of primary small cell carcinoma of the esophagus. Chin Med J (Engl) 2007;120:355-8.

17. Zhang BH, Yang WJ, Zhao L, et al. Surgical treatment and prognostic analysis of 109 patients with primary esophageal small cell carcinoma. Zhonghua Zhong Liu Za Zhi 2012;34:698-702.

18. Situ D, Lin Y, Long H, et al. Surgical treatment for limited-stage primary small cell cancer of the esophagus. Ann Thorac Surg 2013;95:1057-62.

19. Lin EW, Karakasheva TA, Hicks PD, et al. The tumor microenvironment in esophageal cancer. Oncogene 2016;35:5337-49.

20. Mantovani A, Allavena P, Sica A, et al. Cancer-related inflammation. Nature 2008;454:436-44.

21. Suzuki R, Lin SH, Wei X, et al. Prognostic significance of pretreatment total lymphocyte count and neutrophil-tolymphocyte ratio in extensive-stage small-cell lung cancer. Radiother Oncol 2018;126:499-505.

22. Toyokawa T, Kubo N, Tamura T, et al. The pretreatment Controlling Nutritional Status (CONUT) score is an independent prognostic factor in patients with resectable thoracic esophageal squamous cell carcinoma: results from a retrospective study. BMC Cancer 2016;16:722-32.

23. Chan JC, Chan DL, Diakos CI, et al. The lymphocyte-tomonocyte ratio is a superior predictor of overall survival in comparison to established biomarkers of resectable colorectal cancer. Ann Surg 2017;265:539-46.

24. Xie MR, Xu SB, Sun XH, et al. Role of surgery in the management and prognosis of limited-stage small cell carcinoma of the esophagus. Dis Esophagus 2015;28:476-82.

25. Lu XJ, Luo JD, Ling Y, et al. Management of small cell carcinoma of esophagus in China. J Gastrointest Surg 2013;17:1181-7.

26. Mariette C, Markar SR, Dabakuyo-Yonli TS, et al. Hybrid Minimally Invasive Esophagectomy for Esophageal Cancer. N Engl J Med 2019;380:152-62.

27. Xu L, Li Y, Liu X, et al. Treatment Strategies and Prognostic Factors of Limited-Stage Primary Small Cell Carcinoma of the Esophagus. J Thorac Oncol 2017;12:1834-44.

28. Zou B, Li T, Zhou Q, et al. Adjuvant therapeutic modalities in primary small cell carcinoma of esophagus 
patients: A retrospective cohort study of multicenter clinical outcomes. Medicine (Baltimore) 2016;95:e3507.

29. Wang HH, Zaorsky NG, Meng MB, et al. Multimodality therapy is recommended for limited-stage combined small cell esophageal carcinoma. Onco Targets Ther 2015;8:437-44.

30. Kelsen DP, Weston E, Kurtz R, et al. Small-cell carcinoma of the esophagus: treatment by chemotherapy alone. Cancer 1980;45:1558-61.

31. Meng MB, Zaorsky NG, Jiang C, et al. Radiotherapy and chemotherapy are associated with improved outcomes over surgery and chemotherapy in the management of limitedstage small cell esophageal carcinoma. Radiother Oncol 2013;106:317-22.

32. Raja S, Rice TW, Rajeswaran J, et al. Esophageal small-

Cite this article as: Miao H, Li R, Chen D, Hu J, Chen Y, Wen Z. Survival outcomes and prognostic factors of primary small cell carcinoma of the esophagus. J Thorac Dis 2021;13(5):2790-2802. doi: 10.21037/jtd-20-3334 cell cancer: study of a rare disease. Dis Esophagus 2013;26:690-5.

33. Chen B, Yang H, Ma H, et al. Radiotherapy for small cell carcinoma of the esophagus: outcomes and prognostic factors from a retrospective study. Radiat Oncol 2019;14:210.

34. Pasquali S, Yim G, Vohra RS, et al. Survival After Neoadjuvant and Adjuvant Treatments Compared to Surgery Alone for Resectable Esophageal Carcinoma: A Network Meta-analysis. Ann Surg 2017;265:481-91.

35. Pasquali S, Yim G, Vohra RS, et al. Survival After Neoadjuvant and Adjuvant Treatments Compared to Surgery Alone for Resectable Esophageal Carcinoma: A Network Meta-analysis. Ann Surg 2017;265:481-91. 


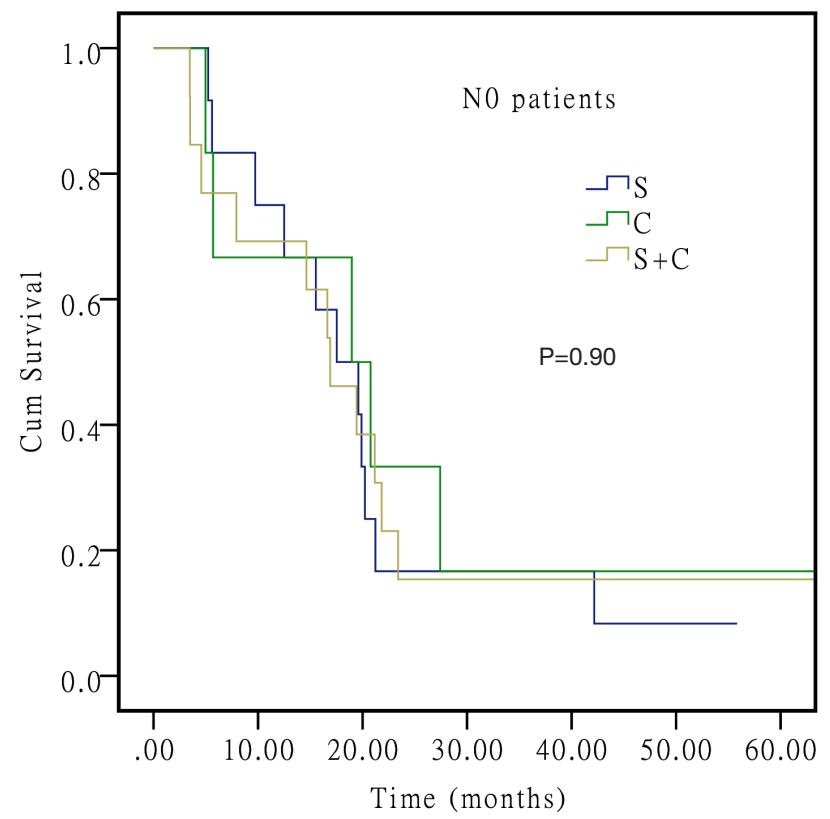

Figure S1 Kaplan-Meier survival curve of OS for N0 patients treated with S/C or S + C. OS, overall survival; S, surgery; C, chemotherapy. 